\title{
A Method for Characterizing Essential Tremor from the Shoulder to the Wrist
}

\author{
Daniel W. Geiger \\ Brigham Young University Mechanical Engineering Department \\ Dennis Eggett \\ Brigham Young University Department of Statistics, theegg@byu.edu \\ Steven K. Charles \\ Brigham Young University Department of Mechanical Engineering and Neuroscience, skcharles@byu.edu
}

Follow this and additional works at: https://scholarsarchive.byu.edu/facpub

Part of the Mechanical Engineering Commons

\section{Original Publication Citation}

D. W. Geiger, D. L. Eggett, and S. K. Charles, "A method for characterizing essential tremor from the shoulder to the wrist," Clinical Biomechanics, vol. 52, pp. 117-123, 2018/02/01/ 2018.

\section{BYU ScholarsArchive Citation}

Geiger, Daniel W.; Eggett, Dennis; and Charles, Steven K., "A Method for Characterizing Essential Tremor from the Shoulder to the Wrist" (2018). Faculty Publications. 2113.

https://scholarsarchive.byu.edu/facpub/2113

This Peer-Reviewed Article is brought to you for free and open access by BYU ScholarsArchive. It has been accepted for inclusion in Faculty Publications by an authorized administrator of BYU ScholarsArchive. For more information, please contact ellen_amatangelo@byu.edu. 
1 Title:

A Method for Characterizing Essential Tremor from the Shoulder to the Wrist

3

4 Authors:

5 Daniel W. Geiger ${ }^{1}$, Dennis Eggett ${ }^{2}$, Steven K. Charles ${ }^{1,3}$

$6 \quad{ }^{1}$ Department of Mechanical Engineering, Brigham Young University, 435 CTB, Provo, UT 84602, United States

$7 \quad{ }^{2}$ Department of Statistics, Brigham Young University, 223 TMCB, Provo, UT 84602, United States

$8 \quad{ }^{3}$ Neuroscience Center, Brigham Younger University, 435 CTB, Provo, UT 84602, United States

9

10 Corresponding Author:

11 Steven Charles

12 Department of Mechanical Engineering

13 Brigham Young University

$14 \quad 435$ CTB, Provo, UT 84602

15 United States

$16 \quad$ (801) 422-7369

17 skcharles@byu.edu

18

19 Running Title:

20 Characterizing Essential Tremor

21

22 Key Words:

23 Essential tremor; upper limb; motor control; inverse kinematics 


\section{Background}

27 Despite the pervasive and devastating effect of Essential Tremor (ET), the distribution of ET

28 throughout the upper limb is unknown. We developed a method for characterizing the distribution

29 of ET and performed a preliminary characterization in a small number of subjects with ET.

Methods

32 Using orientation sensors and inverse kinematics, we measured tremor in each of the seven major 33 degrees of freedom (DOF) from the shoulder to the wrist while ten patients with mild ET assumed 3416 different postures. We described the tremor in each DOF in terms of power spectral density 35 measures and investigated how tremor varied between DOF, postures, gravitational torques, and repetitions.

39 Our method successfully resulted in tremor measures in each DOF, allowing one to compare 40 tremor between DOF and determine the distribution of tremor throughout the upper limb, including 41 how the distribution changes with posture. In our small number of subjects, we found that the 42 amount of power in the frequency band associated with ET (4-12 Hz) was lowest in the shoulder 43 and greatest in the wrist. Similarly, the existence and amplitude of peaks in this band increased 44 from proximal to distal. Although the amount of tremor differed significantly between postures, 45 we did not find any clear patterns with changes in posture or gravitational torque.

\section{Interpretation}

48 This method can be used to characterize the distribution of tremor throughout the upper limb. Our

49 preliminary characterization suggests that the amount of tremor increases in a proximal-distal 50 manner. 


\section{INTRODUCTION}

Essential Tremor (ET) is one of the most common movement disorders [1, 2]. It is described as a visible, persistent, bilateral, largely symmetric postural and kinetic tremor involving mainly the hands and forearms [3]. ET is progressive and adversely affects or limits subjects' ability to perform many common activities of daily living $[4,5]$.

Despite its high prevalence, the mechanical characteristics of ET have not been characterized throughout the upper limb. To clarify, many studies have used accelerometers [6, 7], gyroscopes [8], lasers [9], and EMG [10-14] to quantify mechanical characteristics of ET and uncover important aspects of ET, including the frequency and amplitude [4, 15], dynamics [16, 17], response to loading [18-21], and neural origin of ET [22-24]. However, almost all of these studies have focused on tremor at a single point (e.g. endpoint tremor) or in a single degree of freedom (DOF), often wrist flexion-extension. While this focus was appropriate for isolating specific aspects of ET, we do not know where in the upper limb ET originates mechanically (which muscles or joints), how it propagates, and how it is distributed throughout the upper limb.

Understanding the mechanical origin, propagation, and distribution of ET is important for improving our understanding of ET and developing assistive devices for patients. ET is commonly diagnosed and described through the use of clinical rating scales $[25,26]$ and is often confused with other tremor disorders such as Parkinson's disease [27] or dystonia. Furthermore, ET is at least in part a diagnosis by exclusion, and it has been suggested that ET may actually consist of a number of distinctive subtypes $[28,29]$. A more thorough characterization of ET throughout the upper limb may uncover differences with other tremor disorders and subgroups within ET. In addition, treatment options for patients are limited, and many patients seek a low-cost, lowcommitment alternative to medication and surgery in the form of assistive devices. One might envision a wearable upper limb device (e.g. an orthosis) specifically designed to suppress tremor. Developing such assistive devices requires an understanding of ET throughout the upper limb. As far as we are aware, only one study to-date has investigated the distribution of ET among DOF of the upper limb. Rocon and Pons used gyroscopic sensors to measure the amount of tremor in four DOF (elbow flexion-extension, forearm pronation-supination, wrist flexion-extension, and wrist radial-ulnar deviation) in 21 patients with ET [30]. Among these DOF, tremor amplitude was found to increase proximal-distally, and tremor frequency was found to be similar between DOF. However, tremor distribution has not been characterized throughout the upper limb.

The purpose of this study was to develop a method for characterizing the distribution of ET in all 7 DOF from the shoulder to the wrist (shoulder flexion-extension, abduction-adduction, and internal-external humeral rotation, plus those listed above) and to perform a preliminary characterization in a small number of subjects using this method. Using orientation sensors and inverse kinematics, we estimated the tremor in each DOF and calculated measures describing the amount and frequency of the tremor. Furthermore, we used these measures to investigate how tremor varied between DOF, postures, and levels of gravitational torque to provide a preliminary characterization of ET from the shoulder to the wrist.

\section{METHODS}

\subsection{Subjects}

Ten subjects participated in this study (Table 1). All subjects exhibited tremor in the upper limbs that was not limited to writing tremor and reported that they had been diagnosed with ET by 
a neurologist. Subjects with an age of onset before 20 or after 65 were excluded as early- and lateonset cases [31,32]. Following procedures approved by Brigham Young University's Institutional Review Board, informed consent was obtained from all subjects, after which subjects were evaluated using the Fahn-Tolosa-Marin (FTM) tremor rating scale [33]. The tenth subject had more than half of the statistically significant peaks in tremor power spectra (see below) for all subjects and was excluded from the analysis as an outlier.

\subsection{Experimental Setup}

Subjects were seated on a stool ( $\sim 19$ " in height) with no back, and one of their arms was

106 fitted with four sensors from an electromagnetic motion capture system (trakSTAR by Ascension Technologies, Burlington, VT). The arm that exhibited more severe tremor was tested or, if tremor was reported to be the same in both arms, the subject's dominant arm was tested. Sensors were placed on the dorsum of the hand over the third and fourth metacarpals, the posterior aspect of the forearm just proximal to the wrist, the posterior aspect of the upper arm just proximal to the elbow over the triceps tendon, and over the acromion, straddling the acromial angle for stability. Each sensor was placed in a small, custom-made plastic to minimize rolling and taped in place. The system recorded the orientation of each sensor as three Euler angles with a time-varying sampling rate around 360 samples/s (mean \pm SD of time between samples: $2.77 \pm 0.48 \mathrm{~ms}$ ), a static accuracy of $0.5^{\circ}$, and a resolution of $0.021^{\circ}$. The mass of each sensor, plastic holder and 6 inches of cable was approximately $2.6,2.4$, and 2.59 grams respectively and was assumed to interfere minimally with natural tremor (on average for our subjects, this weight represented 1.1, 0.39 , and $0.23 \%$ of the mass of the hand, forearm, and upper arm, respectively).

The body-fixed coordinate frames of the arm were defined according to the ISB

\subsection{Experimental Procedure}

Each subject placed their upper limb in sixteen different postures (Figure 2), holding each posture for 15 seconds. Once tremor was measured in all 16 postures, we repeated the process for a total of four repetitions. Postures were always assumed in numerical order. All postures were demonstrated to the subject, with special instructions for the following postures. Posture 1 was identical to the calibration posture. For posture 2, subjects were instructed to let their arm hang limp without active elbow extension. For postures 8,15 , and 16, subjects were instructed to move in a specific DOF to the limit of their comfortable range of motion (ROM) in shoulder extension, external humeral rotation, and elbow flexion, respectively. The forearm was pronated in posture 15. For postures 9-14 (wrist flexion, wrist extension, ulnar deviation, radial deviation, forearm pronation, and forearm supination, respectively), subjects were instructed to move from calibration posture in a specific DOF to the limit of their comfortable ROM and then chose a position that felt halfway between the limit and the starting position.

\subsection{Data Processing}

Inverse Kinematics: After resampling the sensor data at $360 \mathrm{~Hz}$ to obtain a constant sampling rate, we derived the joint angles for each DOF of the arm using inverse kinematics. The 
DOF of the upper limb were defined using ISB standards [34] except for the shoulder joint. The ISB standard defines the shoulder DOF using a Y-X'-Y" rotation sequence, which places the calibration posture in gimbal lock. To avoid this issue, we used a $Z-X^{\prime}-Y^{\prime}$ ' rotation sequence based on the coordinate frame defined by ISB. Thus the 7 DOF measured in this study listed in order from proximal to distal are: shoulder flexion/extension $\left(\alpha_{\mathrm{h}}\right)$, abduction/adduction $(\beta \mathrm{h})$, and humeral rotation $\left(\gamma_{\mathrm{h}}\right)$ of the glenohumeral joint; elbow flexion/extension (EFE); forearm pronation/supination (FPS); and flexion/extension (WFE) and radial/ulnar deviation (WRUD) of the wrist. Because we measured motion at the glenohumeral joint instead of the thoracohumeral joint, we refer to $\alpha_{\mathrm{h}}, \beta_{\mathrm{h}}$ and $\gamma_{\mathrm{h}}$ instead of shoulder flexion/extension, abduction/adduction, and humeral rotation. For each subject and posture, we calculated the gravitational torque experienced in each DOF (Appendix).

Measures: Tremor was quantified using four measures as follows. We first transformed all data into the frequency domain. More specifically, we used Welch's Method to estimate the power spectral density of each 15 -sec trial. To reduce windowing artifacts, we removed the mean from the time-series data of each trial before performing Welch's Method. Welch's Method was implemented using Matlab's pwelch function with Hamming windows of 1024 samples $(2.8 \mathrm{sec})$ and $50 \%$ overlap. The first measure represents the power in the frequency band commonly associated with ET (4-12 Hz). It was calculated as the area under the power spectral density curve between 4 and $12 \mathrm{~Hz}$ using the trapezoidal method. All trials with power beyond 3 standard deviations from the mean were considered outliers and excluded from further analysis. The remaining measures describe the peaks in the power spectra. We used a sliding-window constantfalse-alarm-rate peak detection algorithm [35] using a $1 \mathrm{~Hz}$ window and $1.5 \mathrm{~Hz}$ sidebands to determine the existence of peaks over the $4-12 \mathrm{~Hz}$ frequency band. This method uses a statistical approach to determine peaks as those maxima that can be considered outliers compared to the surrounding data. More specifically, it compares the height of the maximum in a moving window to the mean and standard deviation of the data in the sidebands surrounding the window. Maxima are considered outliers and therefore peaks if they are a sufficient number of standard deviations above the mean. It is common practice in statistical analysis to identify outliers as those samples that are more than 2-3 standard deviations above the mean. We chose the more conservative value of 3 standard deviations because it identified the same peaks we observed by visual inspection. If multiple peaks existed, we focused on the peak with the greatest statistical significance (i.e. the greatest number of standard deviations from the mean). The last three measures are peak existence (binary measure indicating whether a given power spectrum exhibited a peak) and, if a peak existed, the frequency and amplitude of that peak.

\subsection{Data Analysis}

The mean and standard deviation of joint angles in different postures were calculated using circular statistics [36]. We performed statistical analyses to determine the effects of various factors (DOF, posture, repetition, and gravitational torque) on the tremor measures (power and peak existence, frequency, and amplitude). To determine the effect of DOF, posture, and repetition on each measure, we performed separately for each measure a mixed-model ANOVA with DOF, posture, and repetition as fixed factors and subject as a random factor. The effect of gravitational torque on tremor in each DOF was determined by correlating the power in each DOF against the gravitational torque in that DOF. Because subjects exhibited different amounts of tremor, we performed the correlations separately for each subject and averaged the correlation coefficients. 
Typical joint angle data are illustrated in Figure 3. Means and standard deviations of joint angles were calculated for all 16 postures. Angle means were generally within $15^{\circ}$ of expected values (nominal values based on Figure 2). For postures with significant shoulder displacement (postures 3-7), the glenohumeral joint angles $\left(\alpha_{\mathrm{h}}, \beta_{\mathrm{h}}, \gamma_{\mathrm{h}}\right)$ were quite different than the expected thoracohumeral joint angles. In particular, $\beta_{\mathrm{h}}$ was only about $-35^{\circ}$ for postures $3-5$; for postures 6 $7, \alpha_{\mathrm{h}}$ was only about $50^{\circ}$. These differences were likely due to motion of the scapula relative to the thorax [37], movement of the skin over the scapula when the arm is abducted or flexed at $90^{\circ}$, patients' limited ROM, and difficulty in precisely copying the demonstrated postures. Also, postures with full elbow extension (postures $3,5,6$ ) had elbow flexion angles on the order of $30^{\circ}$ instead of zero. This difference is likely due to the elbow carrying angle and our definition of the elbow angle. The elbow carrying angle is usually $5-15^{\circ}$ in men and $10-25^{\circ}$ in women [38], but for simplicity we assumed it to be zero. Also, to allow for a calibration method in which landmarks were aligned in the transverse plane, we used the wrist joint center as the distal landmark on the forearm instead of the ulnar styloid [34], further reducing the likelihood of reducing the elbow flexion-extension angle to zero. In addition, in postures with full elbow extension $(3,5,6), \gamma_{\mathrm{h}}$ and FPS differed from the expected angles, likely because of soft-tissue artifact (known to be high for humeral rotation) and patients' limited ROM. Even with these exceptions, the vast majority of angle means were close to the expected values. Furthermore, our analysis focused on the changes in the angles that occurred over time in a given posture (tremor), not the absolute values of those angles.

The power varied significantly by DOF $(\mathrm{p}<0.0001)$ and was greatest for WFE, lowest for $\beta_{\mathrm{h}}$, and roughly similar for the other DOF (Figure 4). We also found statistically significant differences in power between postures $(\mathrm{p}<0.0001)$, but there were no obvious patterns underlying the differences (Figure 4). We expected similar postures to exhibit similar amounts of tremor, but they did not. For example, although postures 1 and 9-14 are similar (Figure 2), this group includes the posture with the lowest amount of tremor (posture 1) and one of the postures with the highest amount of tremor (posture 9). Other comparisons of similar postures likewise exhibited relatively large differences in tremor. We did not find any significant differences between repetitions for power $(\mathrm{p}=0.67)$. Power was not well correlated with gravitational torque; averaged across subjects, the mean $\mathrm{R}^{2}$ values were below 0.4 for all DOF (and below 0.2 for all DOF except $\gamma_{\mathrm{h}}$ ).

Only about $30 \%$ of observations had peaks in the frequency domain that were significant (as defined in the Methods section). Nevertheless, there were significant differences in the number, amplitude, and frequency of peaks between DOF ( $<0.0001$ in all three cases). The three distalmost DOF (FPS, WFE, and WRUD) had the greatest number of peaks and the tallest peaks, and peak frequencies decreased slightly from proximal to distal (Figure 5).

\section{DISCUSSION}

Despite its pervasive and devastating effect on upper limb function, we do not have a thorough understanding of how ET is distributed throughout the upper limb. This paper presents a method for determining the distribution of tremor in all major DOF from the shoulder to the wrist. This method relies on motion capture and inverse kinematics to measure the angular displacement due to tremor and calculate measures of tremor severity in each DOF. We have tested this method on a small number of subjects with mild ET and present preliminary results.

\subsection{Methodology}


While accelerometers, which measure linear acceleration, are the most common device used to measure tremor, characterizing tremor in each DOF requires inverse kinematics which are more easily performed with measurements of orientation (i.e. angle) instead of linear acceleration. Using orientation avoids problems such as drift, gravitational acceleration, and the influence of sensor location, which are common to accelerometers and complicate the inverse kinematics. Orientation measurements can be obtained through optoelectronic or electromagnetic motion capture systems, which can provide data quality comparable to accelerometry.

Rotations in multiple DOF are inherently more complex than translations in multiple dimensions, and Euler angles are sometimes difficult to interpret. We used ISB standards (with slight modifications for use with our electromagnetic motion capture system-see Methods) for the distal DOF of the upper limb [34], where the axes and order of rotation correspond to anatomical axes and the natural hierarchy of DOF in the limb. However, the ISB standard for the shoulder places neutral shoulder position (anatomical posture) in gimbal lock [38]. Since we used neutral shoulder position for calibration and for many of our postures, using the ISB standard would have resulted in large errors due to gimbal lock, so we used a Z-X'-Y'" rotation order instead.

Measuring the orientation of the skeleton using sensors attached to the skin introduces an error when the skin moves relative to the underlying bones [39]. The error due to such soft-tissue artifacts (STA) is believed to be most pronounced for large displacements from neutral position. Our experiment included many postures that were relatively far from neutral position and likely contributed to errors in the calculated joint angles (see Results). That said, our analysis focused on the changes in the angles that occurred over time, not the absolute values of those angles, so steadystate errors in the absolute values of the joint angles are unlikely to have had a large effect on our tremor measures.

While some studies have investigated how adding weight to the subject's upper limb affects tremor, to our knowledge no studies have considered how the limb's own weight affects tremor or how that effect changes with the orientation of the limb. The algorithm presented here (Appendix) enables calculation of the torque exerted in each DOF due to the weight of all limb segments distal to that DOF for any posture, and it does so using the same sensor data used in the inverse kinematics, so no additional measurements are required.

All of our dependent measures were calculated in the frequency domain using power spectrum estimation (PSE). PSE essentially averages a number of Fourier transforms and reduces the effects of noise on the calculated measures; it is therefore preferred over a simple Fourier transform. One disadvantage of using PSE is that it reduces the resolution in frequency, but measurements lasting $15 \mathrm{sec}$ provided sufficient resolution $(0.35 \mathrm{~Hz})$, which could be made even finer by simply extending the duration of each measurement.

Several minor changes could be made to improve the method presented here. Measuring shoulder tremor with respect to the torso instead of the scapula would increase clinical relevance [37]. The inverse kinematics algorithm assumed a carrying angle of zero. Adjusting this to include mean carrying angles (measured with the elbow fully extended) [38] could improve the accuracy of the inverse kinematics. Adjusting the landmark calibration method recommended by ISB [34] to work for electromagnetic motion captures systems would eliminate the need for the posture calibration method, which requires subjects to adduct the upper arm into the parasagittal plane (difficult for overweight patients) and hold a posture (difficult for patients with moderate-to-severe tremor). 


\subsection{Preliminary Characterization} The main purpose of this paper was to present a method for characterizing the distribution of tremor throughout the upper limb, and to demonstrate the feasibility of this method in a small number of subjects. Thus the data presented here provide only a preliminary characterization of how tremor is distributed. A larger sample is necessary to obtain more conclusive results. Nevertheless, our sample was sufficiently large to identify some statistically significant effects.

We found significant differences in the amount of tremor between DOF. However, the pattern depended slightly on the measure. Power in the tremor band was greatest in WFE, lowest in $\beta_{\mathrm{h}}$, and roughly similar for the other DOF (Figure 4). In contrast, although peak amplitude was also lowest in $\beta_{\mathrm{h}}$, it exhibited a clear proximal-distal increase (Figure 5). ${ }^{1}$ On the one hand, power is a more robust measure of tremor than peak amplitude; power integrates all values in the tremor band of the power spectral density and is independent of the adjustable parameters required for peak detection. On the other hand, the proximal-distal increase in peak amplitude agrees with the findings of Rocon and Pons, who measured tremor in the four distal-most DOF (elbow, forearm, and wrist) [30].

There were no statistically significant differences in power between repetitions. The four repeated measurements were taken over a period of approximately 30 minutes. This finding indicates that the power in subjects' tremor was relatively constant over this time, in harmony with a prior study that found relatively low diurnal variations in ET [40]. This finding also indicates that any fatigue, if present, was too small to produce significant differences in the amount of tremor (as measured by power).

Only about one-third of trials exhibited significant peaks in power spectral density, likely due the mild nature of subjects' tremor (Table 1) and our relatively conservative peak-detection method. Because power spectrum estimation averages the Fourier transforms of multiple segments, it reduces or eliminates peaks that are not robust. Also, the sliding-window constantfalse-alarm-rate peak detection algorithm [35] detects only those peaks that are statistically different from the surrounding power spectrum.

Some postures' tremor power was significantly different from those of other postures $(p<0.0001)$, but no meaningful pattern was discernable, and the cause of these differences between postures is unknown. Because ET is more associated with postural than resting tremor, we hypothesized that tremor would increase with gravitational torque since it is one of the main differences between rest and posture. We were therefore surprised to find that tremor was not well correlated with gravitational torque $\left(\mathrm{R}^{2}<0.4\right.$ for all DOF).

\subsection{Perspectives}

The end goal of this research is to develop the ability to 1) measure a patient's tremor throughout his/her upper limb, 2) determine for that patient the mechanical origin, propagation, and distribution of the tremor, and 3) identify the optimal location (DOF or muscle) to intervene to minimize the impact of his/her tremor on daily life. Intervention may include the use of a tremor suppression orthosis/exoskeleton, sensory stimulation, botulinum toxin, or other methods. Reaching this end goal requires a number of steps. The first step (presented here) is to develop a method to measure tremor throughout the upper limb and quantify its distribution. The second step, determining the origin and propagation of tremor, will require an iterative combination of

\footnotetext{
${ }^{1}$ Note that since the glenohumeral joint is a spherical joint, the three DOF of the glenohumeral joint are equally proximal. Similarly, the wrist joint can be approximated as a universal joint with intersecting axis, so the two DOF of the wrist are essentially equally distal.
} 
324 simulation and experimental studies. One currently unanswered question is whether the 325 mechanical origin of the tremor is the optimal location to intervene to minimize the detrimental 326 effects of tremor. Therefore, the final step is to determine how to use an understanding of the 327 tremor origin and propagation to determine the optimal location to intervene. This step will require 328 a combination of modeling and validation studies. Here we have presented the first step: a method 329 for quantifying the distribution of tremor in the upper limb. Although tremor distribution will no 330 doubt play a role in determining optimal tremor suppression strategies, the other steps must be 331 accomplished before this role becomes clear.

332 As mentioned, a secondary outcome of this research may be the identification of subgroups 333 within ET, which could lead to improved differential diagnosis. However, reliably identifying 334 subgroups would require a very large number of subjects. If markerless motion capture systems 335 (e.g. Microsoft Kinect) continue to improve and reach sufficiently high accuracy, it may be 336 possible in the future to develop methods for characterizing tremor throughout the upper limb that 337 are fast enough for routine clinical use. This could enable the collection of tremor data from a very 338 large number of subjects and allow subgroups to be identified, if they exist. 
340 This study was funded in part by a grant from the Gerontology Program at Brigham Young 341 University. 
1. Louis, E.D. and J.J. Ferreira, How Common Is the Most Common Adult Movement Disorder? Update on the Worldwide Prevalence of Essential Tremor. Movement Disorders, 2010. 25(5): p. 534-541.

2. Hubble, J.P., K.L. Busenbark, and W.C. Koller, ESSENTIAL TREMOR. Clinical Neuropharmacology, 1989. 12(6): p. 453-482.

3. Deuschl, G., et al., Essential tremor and cerebellar dysfunction - Clinical and kinematic analysis of intention tremor. Brain, 2000. 123: p. 1568-1580.

4. Elble, R.J., Essential tremor frequency decreases with time. Neurology, 2000. 55(10): p. 1547-1551.

5. Manto, M., et al., Evaluation of a wearable orthosis and an associated algorithm for tremor suppression. Physiological Measurement, 2007. 28(4): p. 415-425.

6. Widjaja, F., et al., SENSING OF PATHOLOGICAL TREMOR USING SURFACE ELECTROMYOGRAPHY AND ACCELEROMETER FOR REAL-TIME ATTENUATION. Journal of Mechanics in Medicine and Biology, 2011. 11(5): p. 1347-1371.

7. Hossen, A., et al., Discrimination of Parkinsonian tremor from essential tremor using statistical signal characterization of the spectrum of accelerometer signal. Bio-Medical Materials and Engineering, 2013. 23(6): p. 513-531.

8. Belda-Lois, J.M., et al., Controllable mechanical tremor reduction. Assessment of two orthoses. Technology \& Disability, 2007. 19(4): p. 169-178.

9. Carignan, B., J.-F. Daneault, and C. Duval, The organization of upper limb physiological tremor. European Journal of Applied Physiology, 2012. 112(4): p. 1269-1284.

10. Zhang, D.G., et al., Exploring Peripheral Mechanism of Tremor on Neuromusculoskeletal Model: A General Simulation Study. Ieee Transactions on Biomedical Engineering, 2009. 56(10): p. 2359-2369.

11. Timmer, J., et al., Cross-spectral analysis of physiological tremor and muscle activity - I Theory and application to unsynchronized electromyogram. Biological Cybernetics, 1998. 78(5): p. 349-357.

12. Lauk, M., et al., Side-to-side correlation of muscle activity in physiological and pathological human tremors. Clinical Neurophysiology, 1999. 110(10): p. 1774-1783.

13. Raethjen, J., et al., Tremor analysis in two normal cohorts. Clinical Neurophysiology, 2004. 115(9): p. 2151-2156.

14. Breit, S., et al., Long-term EMG recordings differentiate between parkinsonian and essential tremor. Journal of Neurology, 2008. 255(1): p. 103-111.

15. Elble, R.J., et al., FACTORS INFLUENCING THE AMPLITUDE AND FREQUENCY OF ESSENTIAL TREMOR. Movement Disorders, 1994. 9(6): p. 589-596.

16. Gantert, C., J. Honerkamp, and J. Timmer, Analyzing the dynamics of hand tremor timeseries. Biological Cybernetics, 1992. 66(6): p. 479-484.

17. Timmer, J., et al., Characteristics of hand tremor time-series. Biological Cybernetics, 1993. 70(1): p. 75-80.

18. Wenderoth, N. and O. Bock, Load dependence of simulated central tremor. Biological Cybernetics, 1999. 80(4): p. 285-290.

19. Bock, O. and N. Wenderoth, Dependence of peripheral tremor on mechanical perturbations: a modeling study. Biological Cybernetics, 1999. 80(2): p. 103-108. 
20. Heroux, M.E., G. Pari, and K.E. Norman, The effect of inertial loading on wrist postural tremor in essential tremor. Clinical Neurophysiology, 2009. 120(5): p. 1020-1029.

21. Heroux, M.E., G. Pari, and K.E. Norman, The effect of inertial loading on wrist kinetic tremor and rhythmic muscle activity in individuals with essential tremor. Clinical Neurophysiology, 2011. 122(9): p. 1794-1801.

22. Sommerlade, L., et al., On the estimation of the direction of information flow in networks of dynamical systems. Journal of Neuroscience Methods, 2011. 196(1): p. 182-189.

23. Schelter, B., et al., Testing for directed influences among neural signals using partial directed coherence. Journal of Neuroscience Methods, 2006. 152(1-2): p. 210-219.

24. Elble, R.J., C. Higgins, and C.J. Moody, Stretch reflex oscillations and essential tremor. Journal of Neurology Neurosurgery and Psychiatry, 1987. 50: p. 691-698.

25. Elble, R.J., et al., Tremor amplitude is logarithmically related to 4-and 5-point tremor rating scales. Brain, 2006. 129: p. 2660-2666.

26. Elble, R., et al., Task Force Report: Scales for Screening and Evaluating Tremor Critique and Recommendations. Movement Disorders, 2013. 28(13): p. 1793-1800.

27. Chouinard, S., E.D. Louis, and S. Fahn, Agreement among movement disorder specialists on the clinical diagnosis of essential tremor. Movement Disorders, 1997. 12(6): p. 973976.

28. Elble, R.J. and G. Deuschl, An update on essential tremor. Current Neurology and Neuroscience Reports, 2009. 9(4): p. 273-277.

29. Zesiewicz, T.A., et al., Overview of essential tremor. Neuropsychiatric Disease and Treatment, 2010. 6: p. 401-408.

30. Rocon, E. and J. Pons, Exoskeletons in rehabilitation robotics: Tremor suppression. Springer tracts in advanced robotics, ed. B. Siciliano, O. Khatib, and F. Groen. 2011, Berlin: Springer-Verlag.

31. Louis, E.D. and R. Ottman, Study of possible factors associated with age of onset in essential tremor. Movement Disorders, 2006. 21(11): p. 1980-1986.

32. Louis, E.D., et al., Older Onset Essential Tremor: More Rapid Progression and More Degenerative Pathology. Movement Disorders, 2009. 24(11): p. 1606-1612.

33. Fahn, S., E. Tolosa, and C. Marin, Clinical Rating Scale for Tremor, in Parkinson's Disease and Movment Disorders, J. Jankovic and E. Tolosa, Editors. 1988, Urban \& Schwarzenberg: Baltimore-Munich. p. 225-234.

34. $\mathrm{Wu}, \mathrm{G}$., et al., ISB recommendation on definitions of joint coordinate systems of various joints for the reporting of human joint motion - Part II: shoulder, elbow, wrist and hand. Journal of Biomechanics, 2005. 38(5): p. 981-992.

35. McDonough, R.N. and A.D. Whalen, Detection of Signals in Noise. 2nd ed. 1995, San Diego, CA: Academic Press.

36. Berens, P., CircStat: A MATLAB Toolbox for Circular Statistics. Journal of Statistical Software, 2009. 31(10): p. 1-21.

37. van Andel, C.J., et al., Complete 3D kinematics of upper extremity functional tasks. Gait \& Posture, 2008. 27(1): p. 120-127.

38. Anglin, C. and U.P. Wyss, Review of arm motion analyses. Proceedings of the Institution of Mechanical Engineers Part H-Journal of Engineering in Medicine, 2000. 214(H5): p. 541-555.

39. Cao, L., T. Masuda, and S. Morita, Compensation for the Effect of Soft Tissue Artifact on Humeral Axis Rotation Angle. J Med Dent Sci, 2007. 54. 
433 40. Mostile, G., et al., Amplitude fluctuations in essential tremor. Parkinsonism \& Related 434 Disorders, 2012. 18(7): p. 859-863.

435 
436

437

438

439

440

441

442

\section{TABLES}

\section{Table 1. Subject Data}

\begin{tabular}{c|c|c|c|c|c|c|c|c} 
Subject & Age & $\begin{array}{c}\text { Age of } \\
\text { Onset }\end{array}$ & Gender & $\begin{array}{c}\text { Height } \\
\text { (inches) }\end{array}$ & $\begin{array}{c}\text { Weight } \\
\text { (lbs) }\end{array}$ & BMI & $\begin{array}{c}\text { FTM } \\
\text { Score }\end{array}$ & $\begin{array}{c}\text { Using } \\
\text { Medication }\end{array}$ \\
\hline 1 & 58 & 25 & $\mathrm{M}$ & 68 & 150 & 22.8 & $11.1 \%$ & $\mathrm{Y}$ \\
\hline 2 & 74 & 60 & $\mathrm{~F}$ & 62 & 118 & 21.6 & $11.1 \%$ & $\mathrm{~N}$ \\
\hline 3 & 31 & 25 & $\mathrm{M}$ & 76 & 245 & 29.8 & $13.2 \%$ & $\mathrm{~N}$ \\
\hline 4 & 50 & 22 & $\mathrm{~F}$ & 64 & 142 & 24.4 & $17.7 \%$ & $\mathrm{~N}$ \\
\hline 5 & 75 & 62 & $\mathrm{M}$ & 69 & 188 & 27.8 & $19.4 \%$ & $\mathrm{Y}$ \\
\hline 6 & 53 & 20 & $\mathrm{~F}$ & 63 & 160 & 28.3 & $24.0 \%$ & $\mathrm{Y}$ \\
\hline 7 & 82 & 55 & $\mathrm{M}$ & 65.5 & 170 & 27.9 & $25.0 \%$ & $\mathrm{Y}$ \\
\hline 8 & 62 & 59 & $\mathrm{~F}$ & 66 & 272 & 43.9 & $28.8 \%$ & $\mathrm{Y}$ \\
\hline 9 & 78 & 55 & $\mathrm{~F}$ & 64 & 151 & 25.9 & $31.3 \%$ & $\mathrm{Y}$ \\
\hline Mean & 63.0 & 42.6 & - & 66.4 & 177.3 & 28.0 & $20.2 \%$ & - \\
\hline St. Dev. & 16 & 18.7 & - & 4.3 & 50.3 & 6.5 & $7.5 \%$ & -
\end{tabular}



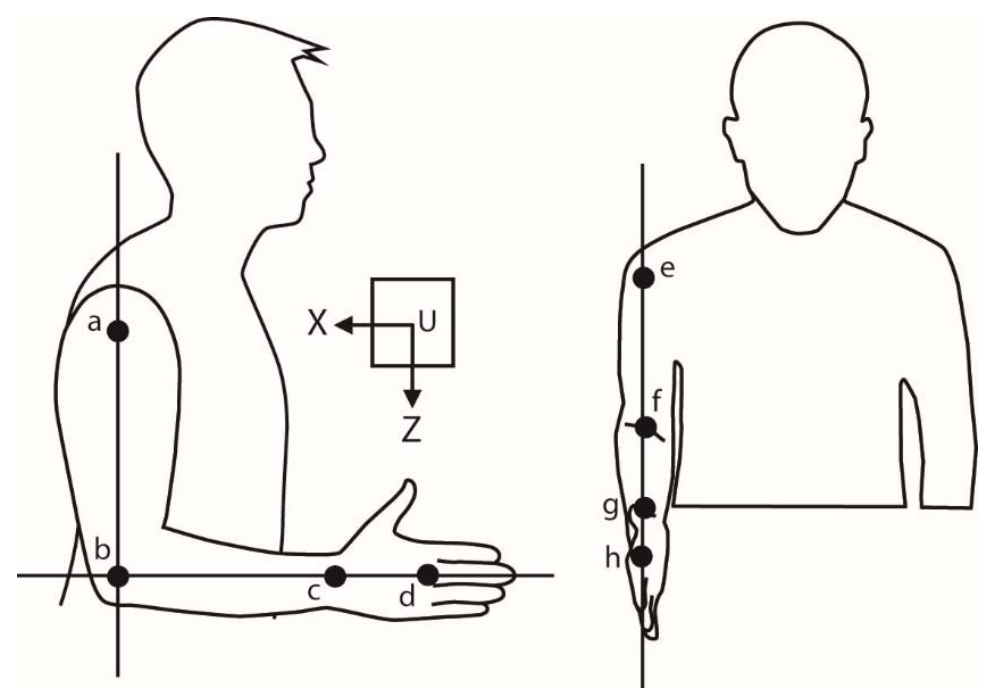

Figure 1. Calibration posture. During calibration, subjects were seated such that their sagittal, frontal, and transverse planes were parallel with the planes of the universal frame of the transmitter of the electromagnetic motion capture system. Subjects assumed the calibration posture shown by aligning 8 landmarks (a-h) in three planes using laser levels. More specifically, in calibration posture, the glenohumeral joint ( $\mathrm{a}$; defined here as the point that was equidistant from the acromion and the ventral and dorsal surfaces of the upper arm, and midway between the medial and lateral surfaces of the upper arm) and lateral epicondyle (b) were in the frontal plane; the lateral epicondyle (b), wrist joint center (c; defined midway between the radial and ulnar styloids), and center of the distal base of the third metacarpal (d) were in the transverse plane; and the glenohumeral joint (e), midpoint between the lateral and medial epicondyles (f) (marked in the 


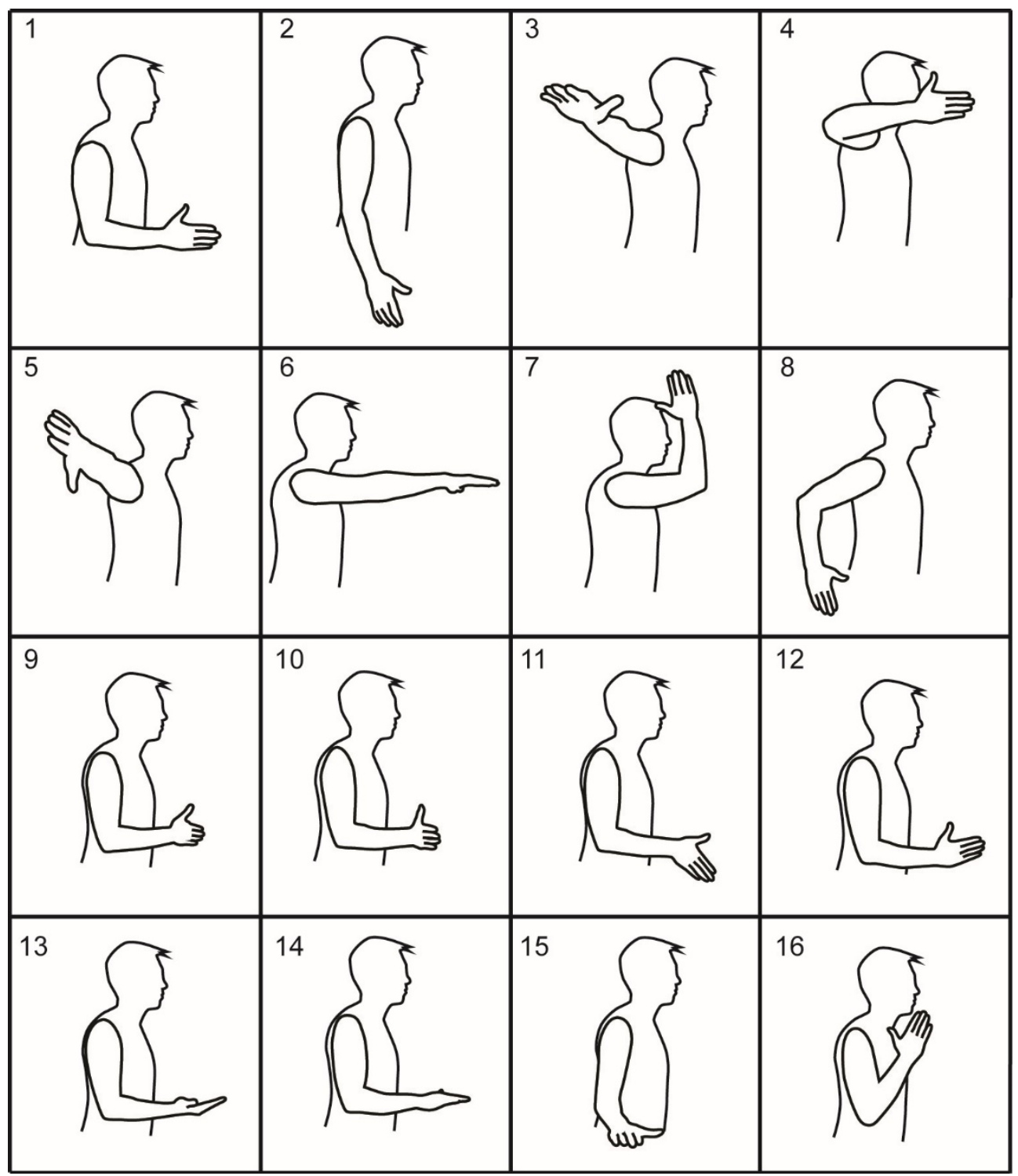

461

462

463

Figure 2. Tremor was measured in each of these 16 postures. 

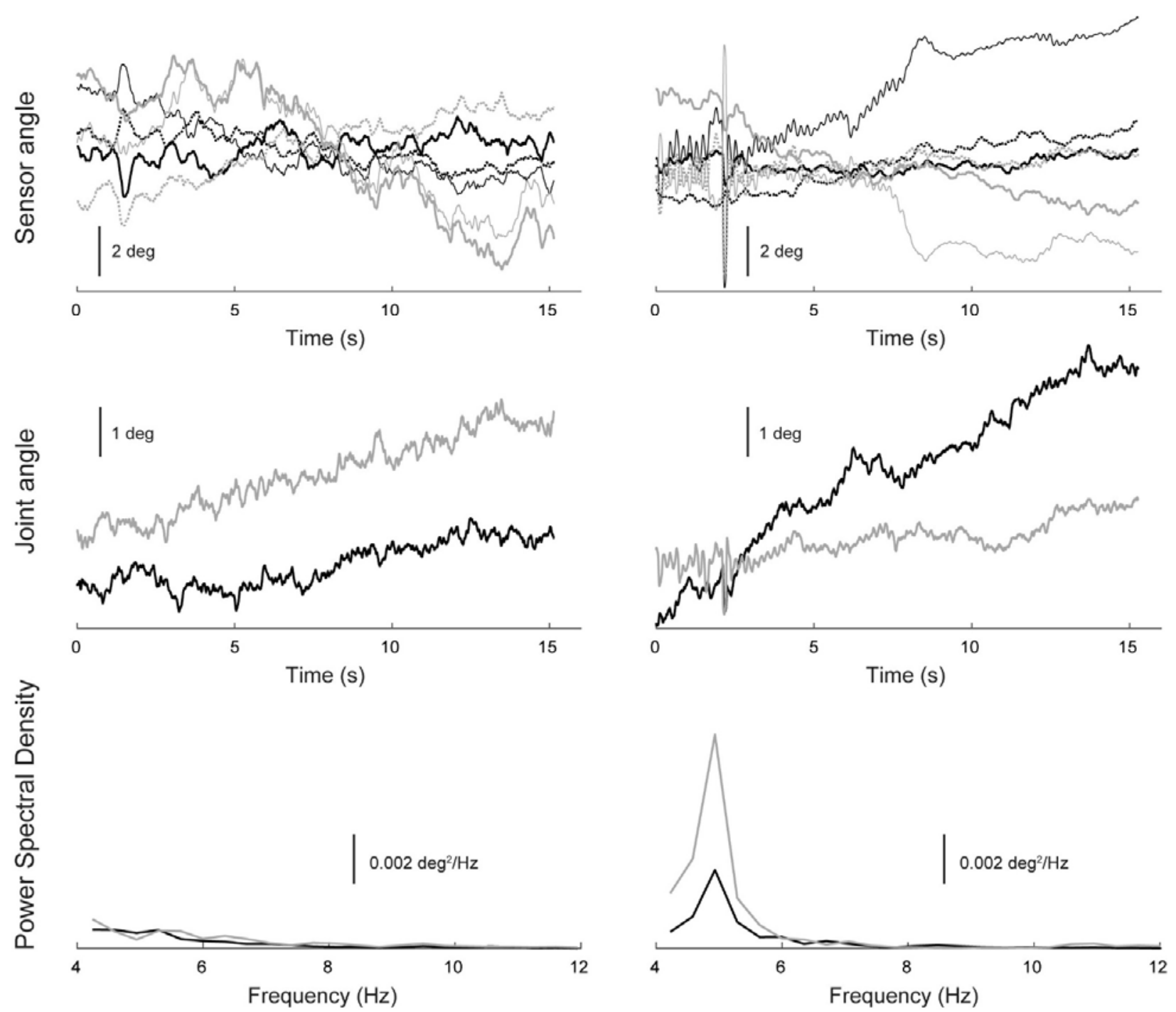

Figure 3. Example data showing the processing procedure from raw sensor angles (top row) via inverse kinematics algorithms to joint angles (middle row), from which we calculated the power spectral density (PSD) (bottom row), where all measures were calculated. The left and right columns show data without and with a clear peak in the tremor band (4-12 Hz) of PSD, respectively. The angles in the top row represent the azimuth (thick solid), elevation (dotted), and roll (thin solid) of the sensors mounted on the forearm (black) and hand (gray). The joint angles and PSD in the middle and bottom rows represent wrist flexion-extension (black) and radial-ulnar 472 deviation (gray). 

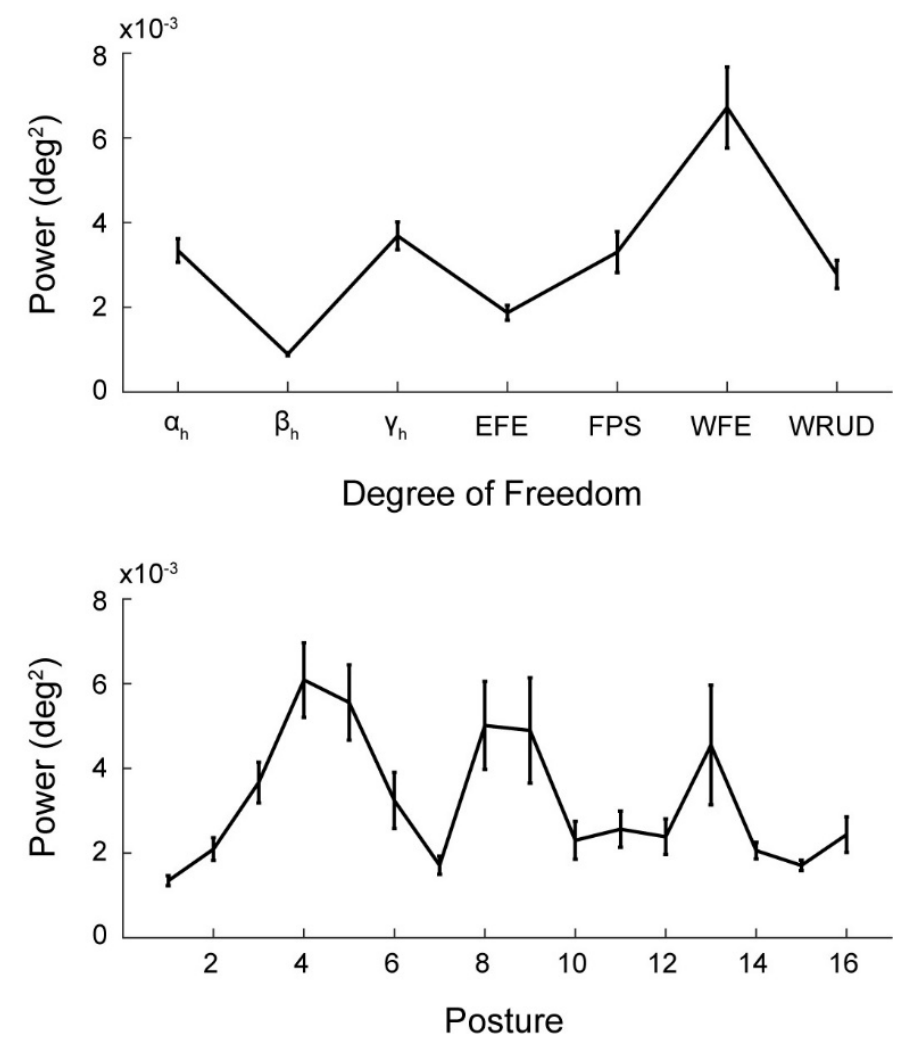

474 475 476 477 478
Figure 4. Power in the tremor band by degree of freedom (DOF; top) and posture (bottom). Postures are shown in Figure 2. The lines and error bars represent the mean and standard error, averaged across all trials in a DOF or posture. 

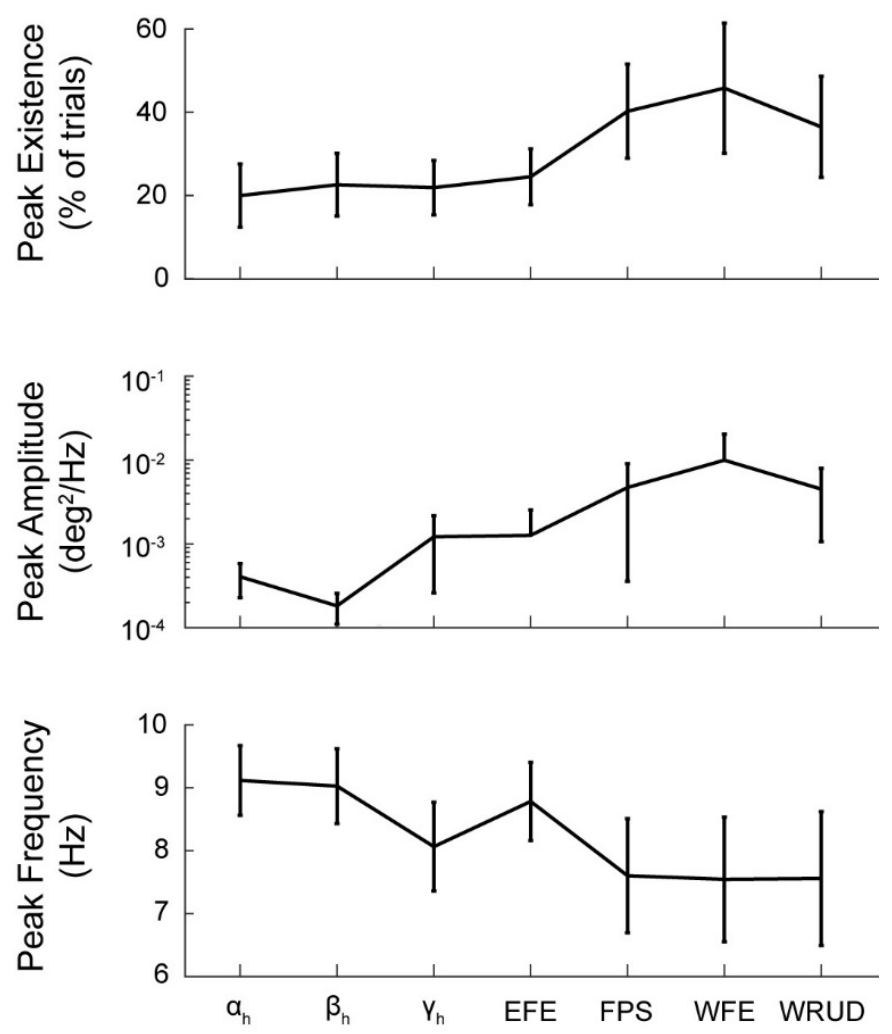

Degree of Freedom

Figure 5. Existence (top), amplitude (middle), and frequency (bottom) of peaks in the tremor band of the power spectral density, separated by degree of freedom. The lines and error bars represent the mean and standard error, averaged across subjects. 